\title{
Multiple Grade Lamination Wound Core: A Novel Technique for Transformer Iron Loss Minimization Using Simulated Annealing With Restarts and an Anisotropy Model
}

\author{
Themistoklis D. Kefalas ${ }^{1}$, Pavlos S. Georgilakis ${ }^{2}$, Antonios G. Kladas ${ }^{1}$, Athanassios T. Souflaris ${ }^{3}$, and \\ Dimitris G. Paparigas ${ }^{3}$ \\ ${ }^{1}$ Faculty of Electrical and Computer Engineering, National Technical University of Athens, GR-15780 Athens, Greece \\ ${ }^{2}$ Department of Production Engineering and Management, Technical University of Crete, GR-73100 Chania, Greece \\ ${ }^{3}$ Schneider Electric AE, GR-32011 Inofyta, Viotia, Greece
}

\begin{abstract}
This paper introduces a novel technique for iron loss minimization of wound core transformers. The proposed technique involves the evaluation of appropriate design variables of wound cores constructed by a combination of standard and high magnetization grade steel. The evaluation of the optimum design variables of the multiple grade lamination wound core is achieved by combining a permeability tensor finite-element model and simulated annealing with restarts.
\end{abstract}

Index Terms-Finite-element methods, magnetic anisotropy, magnetic cores, magnetic losses, optimization methods, power transformers, simulated annealing.

\section{INTRODUCTION}

$\mathbf{T}$ HE present value (PV) of the future iron losses of a typical wound core transformer constitutes more than $60 \%$ of the PV of its total future losses [1]. Any attempt to reduce the iron losses by conventional design optimization methods [2], [3], results in an increase of the transformer's first cost, as of the various materials required to manufacture a transformer, the electrical steel comprises the largest investment [2]. Based on experimental evidence concerning the nonuniformity of the wound core's flux density distribution [4], the transformer's first cost and PV of future iron losses can be reduced effectively, by using wound cores constructed with a combination of standard and high magnetization grade steel.

In order to evaluate the optimum design parameters of a multiple grade lamination wound core, the accurate computation of its flux density distribution and iron losses is needed. Furthermore, an optimization problem such as this presents multiple optima in the feasible domain. We have addressed these two problems by combining a permeability tensor finite-element (FE) model of very low computational cost, as it is based on a particular magnetic scalar potential (MSP) formulation [5], with three stochastic optimization algorithms. From the considered optimization algorithms, an improvement of the simulated annealing (SA) for continuous problems, the simulated annealing with restarts (SAR) [6], is proven to be the most effective in the solution of the specific optimization problem. The multiple grade lamination wound core technique introduces only two design variables, it can be applied after the transformer's design optimization, or it can be integrated directly in the design optimization scheme, resulting in this way in its generalization.

\section{Permeability Tensor FE Model}

According to [4], due to the laminated material, and the geometry of a wound core, the magnitude of the flux density in its

Digital Object Identifier 10.1109/TMAG.2007.916019 outer and most inner steel sheets is lower, than the mean magnitude. Thus, by using standard magnetization and loss grade steel for the outer and inner part, and high magnetization, low loss grade steel for the rest part of the core, it is possible to reduce the wound core first cost and iron loss. By considering the iron-laminated material as homogeneous and anisotropic media at the level of finite elements an accurate representation of the core material is achieved. An elliptic anisotropy model is best suited for the wound core transformer in contrast with the stack core transformer [7], [8]. The specific model is based on the assumption that the field intensity $\mathbf{H}$ has an elliptic trajectory for the modulus of the flux density constant. Therefore, if $\mu_{p}$ and $\mu_{q}$ are the magnetic permeability tangential and normal to the lamination rolling direction, and $r$ is the ratio of the ellipse semi-axes then

$$
\mu_{q}=r \mu_{p}, \quad 0<r<1 .
$$

The permeability tensor $\boldsymbol{\mu}$ in the global coordinate system is given by (2), where $\mathbf{R}$ is the rotation matrix, and $\boldsymbol{\mu}_{F}$ is the permeability tensor in the local coordinate system:

$$
\boldsymbol{\mu}=\mathbf{R}^{-1} \boldsymbol{\mu}_{F} \mathbf{R} .
$$

The specific FE model is going to provide the solution to a stochastic optimization algorithm searching iteratively the optimum design parameters. Therefore, an appropriate formulation must be sought in order to maintain the computational cost within reasonable limits. MSP formulations are advantageous in terms of computational cost, comparing to $\mathrm{FE}$ methods based on the magnetic vector potential (MVP), as there is only one unknown at each node of the FE mesh [5]. Thus, the general potential (GP) formulation [9], a MSP formulation that united and extended early MSP formulations, is usually used. According to GP, the field intensity $\mathbf{H}$ is sought in the following form

$$
\mathbf{H}=\mathbf{H}_{g}+\nabla \Phi_{g}
$$


where $\mathbf{H}_{g}$ is an initial guess magnetic field and $\Phi_{g}$ is the general scalar potential. If $\mathbf{H}_{g}$ satisfies specific conditions [9], then the solution of the problem can be found according to

$$
\nabla \cdot\left(\boldsymbol{\mu} \cdot\left(\mathbf{H}_{g}+\nabla \Phi_{g}\right)\right)=0 .
$$

The evaluation of a suitable $\mathbf{H}_{g}$ is based on a three-step scheme [9]. However, the complexity of the specific three-step procedure as well as the fact that during a nonlinear analysis several iterations are required during the first and third step, results in considerable computational effort and time.

In this paper, a particular scalar potential formulation is adopted, necessitating no prior source field calculation [5]. According to the specific formulation, $\mathbf{H}$ is partitioned as follows:

$$
\mathbf{H}=\mathbf{K}-\nabla \Phi
$$

where $\Phi$ is a scalar potential extended all over the solution domain, and $\mathbf{K}$ is a fictitious field distribution satisfying three conditions which enables to simulate the coil domain [5]. The distribution of $\mathbf{K}$ is easily determined analytically or numerically by the conductors shape [5]. The problem's solution is obtained by discretizing (6) that ensures the total field solenoidality

$$
\nabla \cdot(\boldsymbol{\mu} \cdot(\mathbf{K}-\nabla \Phi))=0
$$

The specific formulation does not suffer from cancellation errors and it is applicable to multiply connected iron regions. Simplicity and computational efficiency are its main advantages, rendering the specific scalar formulation ideal for providing the solution to the optimization problem under consideration. The accurate evaluation of the flux density distribution with the specific FE method is used in conjunction with the experimentally determined specific core losses, for the evaluation of the wound core iron loss.

\section{OptimizATION Methodology}

\section{A. Applying Multiple Grade Lamination Technique After the Transformer's Design Optimization}

This case is not only of practical importance, but it also allows us to study the effect of the multiple grade lamination technique thoroughly as it is isolated from the design optimization procedure. The wound core design variables $x_{3}, x_{4}, x_{5}$, and $x_{6}$, shown in Fig. 1, the magnitude of the mean flux density $B$, and the number of turns of the primary and secondary windings $N_{p, s}$, are constants, predetermined by a typical industrial design optimization procedure [2], [3]. The evaluation of the multiple grade lamination wound core design parameters consists in the minimization of an objective function $f(\mathbf{x})$, where $\mathbf{x}$ is the vector of the core variables $\left(x_{1}, x_{2}\right)$ illustrated in Fig. 1 . The design variables are subject to the following constraints:

$$
0 \leq x_{1} \leq x_{3}, \quad 0 \leq x_{2} \leq x_{3}, \quad 0 \leq x_{1}+x_{2} \leq x_{3} .
$$

The objective function has to take into account the PV of the future iron losses and the cost of the standard and high magneti-

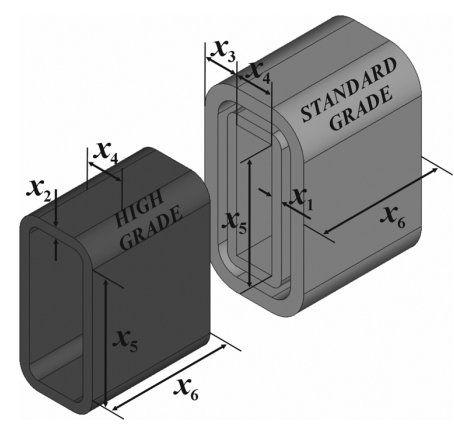

Fig. 1. Representation of the multiple grade lamination wound core variables.

zation grade steel. During the optimization process, the iron loss value is calculated with the use of the FE anisotropy model described in Section II. The mass of the high and standard magnetization material, $M_{\mathrm{HM}}$ and $M_{\mathrm{SM}}$, are calculated by (8) and (9) respectively, where $d_{m s}$ is the magnetic steel density $\left(\mathrm{kg} / \mathrm{m}^{3}\right)$, and $c_{\mathrm{sf}}$ is the wound core empirical stacking factor

$$
\begin{gathered}
M_{\mathrm{HM}}=d_{m s} c_{\mathrm{sf}}\left\{\pi x_{2}^{2} x_{6}+2 x_{2} x_{6}\left(\pi x_{1}+x_{4}+x_{5}\right)\right\} \\
M_{\mathrm{SM}}=d_{m s} c_{\mathrm{sf}}\left\{\pi x_{6}\left(x_{3}^{2}-x_{2}^{2}-2 x_{1} x_{2}\right)\right. \\
\left.+2 x_{6}\left(x_{3}-x_{2}\right)\left(x_{4}+x_{5}\right)\right\} .
\end{gathered}
$$

The objective function is given by (10), where $C_{\mathrm{HM}}$ and $C_{\mathrm{SM}}$ are the high and standard magnetization steel cost $(\$ / \mathrm{kg})$ respectively, $\mathrm{SM}$ is the sales margin, $P_{\mathrm{IL}}$ is the iron losses (W), and $A_{\text {factor }}(\$ / \mathrm{W})$ is the $\mathrm{PV}$ of $1 \mathrm{~W}$ of iron loss over the transformer lifetime [1]

$$
f(\mathbf{x})=\left(C_{\mathrm{HM}} M_{\mathrm{HM}}+C_{\mathrm{SM}} M_{\mathrm{SM}}\right) / \mathrm{SM}+A_{\mathrm{factor}} P_{\mathrm{IL}} .
$$

\section{B. Integrating Multiple Grade Lamination Technique in the Transformer Design Optimization Procedure}

In this case, the multiple grade lamination technique is integrated in a simple design optimization procedure, of a onephase, core type transformer. The objective function must take into account not only the cost of the magnetic steel and the PV of future iron loss but also the cost of the winding material and the PV of future load loss. The variables $x_{3}, x_{4}, x_{5}, x_{6}$ (shown in Fig. 1), $B$, and $N_{p, s}$, are to be determined. By setting $x_{2}=0\left(x_{1}=0\right)$, and $x_{1}=x_{3}\left(x_{2}=x_{3}\right)$ the problem reduces to the design optimization of a transformer constructed by the standard (high) magnetization material. The optimum design and operational parameters are evaluated by minimizing (11), where $C_{\mathrm{Cu}}$ is the winding material cost $(\$ / \mathrm{kg}), M_{\mathrm{Cu}}$ is the winding material mass, $B_{\text {factor }}(\$ / \mathrm{W})$ is the $\mathrm{PV}$ of $1 \mathrm{~W}$ of load loss over the transformer lifetime [1], and $P_{\mathrm{LL}}$ is the load loss (W). The iron loss value is evaluated using the FE model of Section II, $M_{\mathrm{Cu}}, B_{\text {factor }}$ and $P_{\mathrm{LL}}$ are given by (12), (13), and (14) respectively, where $d_{\mathrm{Cu}}$ is the winding material density $\left(\mathrm{kg} / \mathrm{m}^{3}\right), c_{\mathrm{ff}}$ is the coil fill factor, $P$ is the per-unit load, $\rho$ is the winding material resistivity $(\Omega \cdot \mathrm{m})$, and $J$ is the current density $\left(\mathrm{A} / \mathrm{m}^{2}\right)$.

$$
\begin{aligned}
f(\mathbf{x})= & \left(C_{\mathrm{HM}} M_{\mathrm{HM}}+C_{\mathrm{SM}} M_{\mathrm{SM}}+C_{\mathrm{Cu}} M_{\mathrm{Cu}}\right) / \mathrm{SM} \\
& +A_{\text {factor }} P_{\mathrm{IL}}+B_{\mathrm{factor}} P_{\mathrm{LL}}
\end{aligned}
$$




$$
\begin{aligned}
M_{\mathrm{Cu}} & =d_{\mathrm{Cu}} c_{\mathrm{ff}} x_{4} x_{5}\left(2 x_{3}+\pi x_{4}+2 x_{6}\right) \\
B_{\mathrm{factor}} & =A_{\mathrm{factor}} P^{2} \\
P_{\mathrm{LL}} & =c_{\mathrm{ff}} \rho J^{2} x_{4} x_{5}\left(2 x_{3}+\pi x_{4}+2 x_{6}\right) .
\end{aligned}
$$

The objective function of (11) is subject to five nonlinear constraints. Two equality constraints, (15) and (16), representing the primary induced voltage $\left(E_{p}\right)$ constraint, and the rated power $\left(S_{\text {rated }}\right)$ constraint respectively, and the three inequality constraints of (17), where $f$ is the frequency $(\mathrm{Hz})$, $k$ is the portion of the solid conductor area contributed by the primary winding, and $P_{\mathrm{LL}}^{g}, P_{\mathrm{IL}}^{g}$ are the guaranteed load loss, and iron loss respectively (W), specified by international technical specifications and customer needs. The secondary winding turns are equal to $N_{s}=E_{s} N_{p} / E_{p}$, where $E_{s}$ is the secondary induced voltage

$$
\begin{aligned}
& E_{p}-\sqrt{2} \pi f c_{\mathrm{sf}} N_{p} x_{3} x_{6} B=0 \\
& S_{\text {rated }}-\sqrt{2} \pi f c_{\mathrm{sf}} c_{\mathrm{ff}} k J x_{3} x_{6} x_{4} x_{5} B=0 \\
& P_{\mathrm{LL}}<1.15 P_{\mathrm{LL}}^{g}, P_{\mathrm{IL}}<1.15 P_{\mathrm{IL}}^{g}, P_{\mathrm{LL}}+P_{\mathrm{IL}} \\
& \quad<1.1\left(P_{\mathrm{LL}}^{g}+P_{\mathrm{IL}}^{g}\right) .
\end{aligned}
$$

\section{Optimization Algorithms}

For the solution of the optimization problems presented in Section III, five deterministic and three stochastic optimization algorithms have been tested. The deterministic algorithms used are three gradient-based, the Broydon-Fletcher-GoldfarbShanno (BFGS), the Davidon-Fletcher-Powell (DFP), and the steepest descent, and two nongradient methods, the downhill simplex method, and the pattern search. The three stochastic optimization algorithms used are the genetic algorithm (GA), the simulated annealing (SA), and the simulated annealing with restarts (SAR). For continuous problems, e.g., optimization of electromagnetic devices, SA uses a modification of the downhill simplex method to generate random changes. However, premature convergence has been observed [6] that results in the pinning of the simplex in a local minimum. SA then uses a large number of evaluations to explore a small portion of the design space. SAR effectively reduces the objective function evaluations, without revolutionizing SA [10], by forcing the simplex to start from a random point in the space, if it becomes too small [6].

\section{RESULTS AND DiscUSSION}

\section{A. Experimental Verification of the FE Anisotropy Model}

Table I presents a comparison between the calculated iron loss with the FE model of Section II and the respective experimental values. As can be seen from Table I, the computed results are in good agreement with the measured ones and the error is less than $5 \%$ in all cases.

\section{B. Application of the Multiple Grade Lamination Technique}

The GA, SA, and SAR algorithms were tested by minimizing (10) and (11) for a number of wound cores of various geometric and operating parameters. The performance criteria used is the number of objective function evaluations and the ability of the optimization algorithm to find the global minimum. Table II
TABLE I

Calculated Versus Experimental Iron Loss Values $\left(x_{3}=24.3 \mathrm{~mm}\right)$

\begin{tabular}{cccc}
\hline \hline$x_{1}(\mathrm{~mm})$ & $x_{2}(\mathrm{~mm})$ & $\begin{array}{c}\text { Iron loss }(\mathrm{W}) \\
\text { FE anisotropy model }\end{array}$ & $\begin{array}{c}\text { Iron loss }(\mathrm{W}) \\
\text { Experiment }\end{array}$ \\
\hline 24.3 & 0 & 22.7 & 23.1 \\
0 & 24.3 & 18.5 & 19.4 \\
3 & 9 & 21.9 & 22.4 \\
\hline \hline
\end{tabular}

TABLE II

Stochastic Algorithms Convergence Properties

\begin{tabular}{ccccc}
\hline \hline Stochastic algorithm & $x_{1}(\mathrm{~mm})$ & $x_{2}(\mathrm{~mm})$ & $f(\mathbf{x})(\$)$ & $\begin{array}{c}\text { Objective } \\
\text { function calls }\end{array}$ \\
\hline GA & 13.9 & 4.67 & 691.2 & 2,435 \\
SA & 5.91 & 6.68 & 677.5 & 2,597 \\
SAR & 5.91 & 6.70 & 677.5 & 2,080 \\
\hline \hline
\end{tabular}

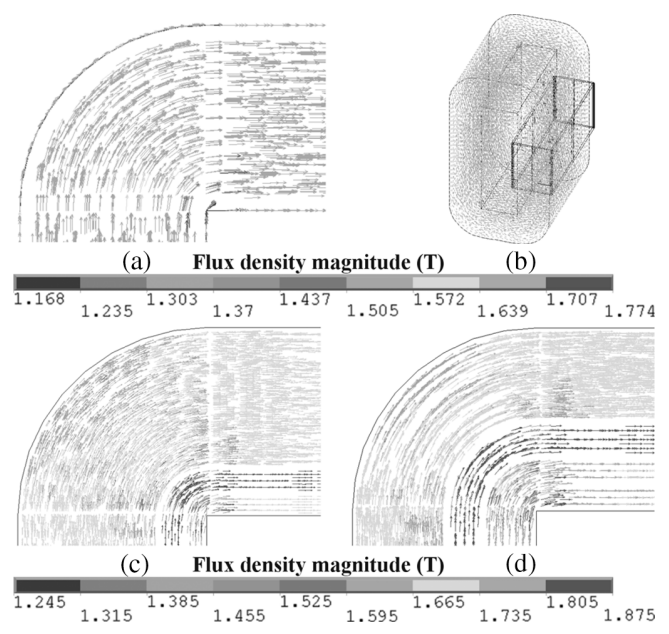

Fig. 2. Flux density distribution, (a) and (b) of the wound core constructed with M4, of the multiple grade lamination wound core with (c) optimum design variables and (d) design variables corresponding to local minima.

summarizes the results obtained from the minimization of (10) by the GA, SA, and SAR algorithms and the number of objective function evaluations. The considered wound core is an outer core, of a $100 \mathrm{kVA}, 20 \mathrm{kV} / 0.4 \mathrm{kV}$ three-phase wound core distribution transformer. The standard and high magnetization materials used are M4 $0.27 \mathrm{~mm}$, and $\mathrm{M}-\mathrm{OH} 0.27 \mathrm{~mm}$ respectively. The geometry parameters of the wound core are $x_{3}=51.3 \mathrm{~mm}$, $x_{4}=57 \mathrm{~mm}, x_{5}=183 \mathrm{~mm}$, and $x_{6}=190 \mathrm{~mm}$, and the mean flux density is $1.723 \mathrm{~T}$.

We have identified a number of local minimums with values very close to the global minimum. Due to this fact the number of successes of the GA was lower than that of the SA and SAR algorithms, as the GA was trapped most of the times in a local minimum. The optimum distribution of the design parameters as calculated by the SA and SAR algorithms correspond to an objective function value of $677.5 \$$, i.e., a $3.2 \%$ reduction of the sum of magnetic steel cost and PV of future iron loss as it was pre-evaluated by a typical industrial design optimization scheme [2]. However the SAR algorithm exhibits a $20 \%$ reduction in objective function calls comparing to the classic SA algorithm. Fig. 2(a) and (b) illustrates the flux density distribution of the 


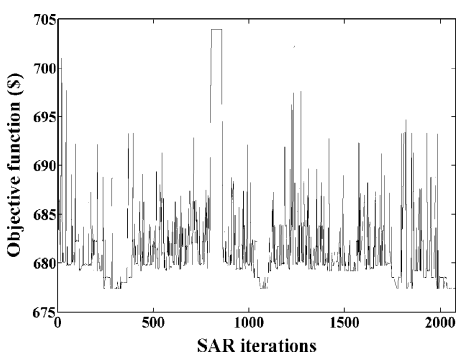

Fig. 3. Variation of the objective function with respect to SAR iterations.

TABLE III

Optimum Designs of A TRANSFormer With DifFERENT CoRe Materials

\begin{tabular}{ccc}
\hline \hline M4 $0.27 \mathrm{~mm}$ & M-OH $0.27 \mathrm{~mm}$ & $\begin{array}{r}\text { Multiple grade, M4 and } \\
\text { M-OH, wound core }\end{array}$ \\
\hline$f=50 \mathrm{~Hz}, J=3 \cdot 10^{6} \mathrm{~A} / \mathrm{m}^{2}, P_{L L}^{g}=560 \mathrm{~W}, P_{I L}^{g}=130 \mathrm{~W}, x_{1} \div x_{6}(\mathrm{~mm})$ \\
\hline$x_{1}=107.6, x_{2}=0$ & $x_{1}=0, x_{2}=113.1$ & $x_{1}=4.2, x_{2}=64.2$ \\
$x_{3}=107.6, x_{4}=60$ & $x_{3}=113.1, x_{4}=49.5$ & $x_{3}=110.8, x_{4}=66$ \\
$x_{5}=268, x_{6}=152$ & $x_{5}=300, x_{6}=152$ & $x_{5}=231, x_{6}=152$ \\
$N_{\mathrm{s}}=3,377, N_{\mathrm{p}}=39$ & $N_{\mathrm{s}}=3,117, N_{\mathrm{p}}=36$ & $N_{\mathrm{s}}=3,203, N_{\mathrm{p}}=37$ \\
$B=1.69 \mathrm{~T}$ & $B=1.74 \mathrm{~T}$ & $B=1.73 \mathrm{~T}$ \\
$P_{\mathrm{IL}}=143.82 \mathrm{~W}$ & $P_{\mathrm{IL}}=140.4 \mathrm{~W}$ & $P_{\mathrm{IL}}=133.4 \mathrm{~W}$ \\
$P_{\mathrm{LL}}=609.4 \mathrm{~W}$ & $P_{\mathrm{LL}}=544.3 \mathrm{~W}$ & $P_{\mathrm{LL}}=598.4 \mathrm{~W}$ \\
$M_{\mathrm{SM}}=120.0 \mathrm{~kg}$ & $M_{\mathrm{SM}}=0 \mathrm{~kg}$ & $M_{\mathrm{SM}}=57.9 \mathrm{~kg}$ \\
$M_{\mathrm{HM}}=0 \mathrm{~kg}$ & $M_{\mathrm{HM}}=133.8 \mathrm{~kg}$ & $M_{\mathrm{HM}}=59.2 \mathrm{~kg}$ \\
$M_{\mathrm{Cu}}=35.6 \mathrm{~kg}$ & $M_{\mathrm{Cu}}=31.8 \mathrm{~kg}$ & $M_{\mathrm{Cu}}=34.92 \mathrm{~kg}$ \\
$\Delta f=0 \%$ & $\Delta f=0.5 \%$ & $\Delta f=2.44 \%$ \\
3,057 evaluations & 3,208 evaluations & 4,526 evaluations \\
\hline \hline
\end{tabular}

wound core constructed with the standard magnetization steel M4, while Fig. 2(c) and (d) shows, respectively, the flux density distribution of the multiple grade lamination wound core with the optimum design parameters, and a set of parameters corresponding to a local minimum. Fig. 3 depicts the variation of the objective function with respect to the SAR iterations.

Table III summarizes the optimum configuration and the number of objective function evaluations, obtained from the minimization of (11) by the SAR algorithm, i.e., the most effective of the three stochastic optimization algorithms tested, while satisfying the constraints (15), (16), and (17). The transformer studied, is a $50 \mathrm{kVA}, 20 \mathrm{kV} / 231 \mathrm{~V}$ one-phase, core type transformer. Table III shows that the application of the multiple grade lamination technique results in a reduction ( $\Delta f=2.44 \%$ and $\Delta f=1.94 \%$ respectively) of the first cost and PV of total future losses, compared to the cases where the transformer is manufactured using only the standard (M4) or the high $(\mathrm{M}-\mathrm{OH})$ magnetization grade steel. As a result, by integrating the multiple grade lamination technique in a typical design optimization procedure, the transformer designer can effectively control not only the magnetic steel cost and the iron losses, but also the winding material cost, the load losses, and other design and operational parameters, in contrast with the method given in Section III-A. The deterministic algorithms reported in Section III-C were also used for the minimization of (10) and (11). As expected they did not determine the global optimum but instead they converge to a local minimum near to the starting point.

\section{CONCLUSION}

The paper introduces a novel methodology enabling transformer design optimization by integrating multiple grade laminations in the standard optimization procedure. It is based on a permeability tensor FE model and the optimization algorithm SAR. This method presents important advantages when compared to the multiple grade lamination cores after the standard transformer design optimization process, as it is taking into account the impact of other parameters, such as the winding material cost and load losses. Consequently, the proposed methodology achieves a further reduction of the sum of first cost and present value of future losses.

\section{ACKNOWLEDGMENT}

This work was supported in part by the E.U.-European Social Fund under Research Project 03ED045 and in part by the Greek Ministry of Development-GSRT.

\section{REFERENCES}

[1] S. Y. Merritt and S. D. Chaitkin, "No-load versus load loss," IEEE Ind. Appl. Mag., vol. 9, no. 6, pp. 21-28, Nov.-Dec. 2003.

[2] P. Georgilakis, N. Hatziargyriou, and D. Paparigas, "AI helps reduce transformer iron losses," IEEE Comp. App. Power, vol. 12, no. 4, pp. 41-46, Oct. 1999.

[3] R. A. Jabr, "Application of geometric programming to transformer design," IEEE Trans. Magn., vol. 41, no. 11, pp. 4261-4269, Nov. 2005.

[4] M. Enokizono, T. Todaka, and K. Nakamura, "Flux distribution in a wound core of a single-phase transformer," J. Magn. Magn. Mater., vol. 160, pp. 61-62, 1996.

[5] A. G. Kladas and J. A. Tegopoulos, "A new scalar potential formulation for 3-D magnetostatics necessitating no source field calculation," IEEE Trans. Magn., vol. 28, no. 2, pp. 1103-1106, Mar. 1992.

[6] S. Alfonzetti, E. Dilettoso, and N. Salerno, "Simulated annealing with restarts for the optimization of electromagnetic devices," IEEE Trans. Magn., vol. 42, no. 4, pp. 1115-1118, Apr. 2006.

[7] M. Enokizono and N. Soda, "Finite element analysis of transformer model core with measured reluctivity tensor," IEEE Trans. Magn., vol. 33, no. 5, pp. 4110-4112, Sep. 1997.

[8] H. V. Sande, T. Boonen, I. Podoleanu, F. Henrotte, and K. Hameyer, "Simulation of a three-phase transformer using an improved anisotropy model," IEEE Trans. Magn., vol. 40, no. 2, pp. 850-855, Mar. 2004.

[9] M. Gyimesi, D. Lavers, T. Pawlak, and D. Ostergaard, "Application of the general potential formulation," IEEE Trans. Magn., vol. 29, no. 2, pp. 1345-1347, Mar. 1993

[10] L. S. Shu, S. Y. Ho, and S. J. Ho, "A novel orthogonal simulated annealing algorithm for optimization of electromagnetic problems," IEEE Trans. Magn., vol. 40, no. 4, pp. 1791-1795, Jul. 2004.

Manuscript received June 24, 2007. Corresponding author: T. Kefalas (e-mail: thkefala@central.ntua.gr). 